\title{
Outcomes of brace treatment for Adolescent Idiopathic Scoliosis - factors affecting the results of the treatment
}

\author{
Toru Maruyama*', Yusuke Nakao, Yosuke Kobayashi, Makoto Miura \\ From 10th International Conference on Conservative Management of Spinal Deformities - SOSORT 2013 \\ Annual Meeting \\ Chicago, IL, USA. 8-11 May 2013
}

\section{Background}

Controversies still exist regarding the effectiveness of brace treatment for adolescent idiopathic scoliosis (AIS).

\section{Purpose}

The goal of this study was to analyze the outcomes of brace treatment for AIS and to clarify the factors affecting the results of the treatment.

\section{Methods}

According to the Scoliosis Research Society (SRS) AIS brace studies standardization criteria, the following conditions were used to determine which patients to include in this study: aged 10 years or older, Risser 0-II, primary curve magnitude $25^{\circ}-40^{\circ}$ and no prior treatment when the brace was subscribed. At skeletal maturity, the percentage of patients whose curve progressed more than $6^{\circ}$, whose curve exceeded $45^{\circ}$ or who underwent surgery was investigated. These results were compared with natural history or other brace studies. Factors affecting the results were analyzed.

\section{Results}

A total of 29 patients, 24 females and 5 males, met the SRS inclusion criteria. Average age at the beginning of the treatment was 12.0 years (range 10 to 15 years). Risser sign was 0 in 10, I in 6 and II in 13 patients. Curve pattern was thoracic $(\mathrm{T})$ in 11 , thoracolumbar or lumbar (TL) in 11 and double (D) in 7 patients. Average Cobb angle before treatment was $31.3^{\circ}$. Initial correction rate by the brace was $51.7 \%$ on average $(35.2 \%$ for $\mathrm{T}$,
75.5\% for TL and $43.4 \%$ for D curve). Most patients wore their brace part-time, at home or at night. All the patients were followed until they reached skeletal maturity, with an average follow-up period of 29 months. The average Cobb angle at follow-up was $32.2^{\circ}$. Of 29 patients, six (21\%) progressed more than $6^{\circ}$ and three (10\%) exceeded $45^{\circ}$. Only one patient (3\%) underwent surgical treatment during the study period. These results were more favorable than the reported natural history or most of the other brace studies. Factors predicting the better results of brace treatment were Risser sign of I or II at the beginning of the treatment and better initial correction rate with the brace.

\section{Conclusions and discussion}

Of the curves with AIS, $79 \%$ were stabilized by the treatment. These results illustrate that brace treatment was effective for the treatment of AIS.

Published: 18 September 2013

Reference

1. Richards B Stephens, et al: Standardization of Criteria for Adolescent Idiopathic Scoliosis Brace Studies. Spine 30(18):2068-2075.

doi:10.1186/1748-7161-8-S2-O51

Cite this article as: Maruyama et al: Outcomes of brace treatment for Adolescent Idiopathic Scoliosis - factors affecting the results of the treatment. Scoliosis 2013 8(Suppl 2):051.

* Correspondence: tmaruyama17@yahoo.co.jp

Saitama Medical Center, Saitama Medical University, Saitama, Japan

(c) 2013 Maruyama et al; licensee BioMed Central Ltd. This is an Open Access article distributed under the terms of the Creative 